\title{
A prospective comparison of conventional cytology and digital image analysis for the identification of pancreatic malignancy in patients undergoing EUS-FNA
}

\author{
Fanyang Kong ${ }^{1,2}$, Xiangyu Kong ${ }^{1}$, Jianwei Zhu ${ }^{1}$, Tao Sun ${ }^{3}$, Yiqi Du ${ }^{1}$, Kaixuan Wang ${ }^{1}$, Zhendong Jin ${ }^{1}$, \\ Zhaoshen $\mathrm{Li}^{1,2}$, Dong Wang ${ }^{1,2}$ \\ ${ }^{1}$ Department of Gastroenterology, Gongli Hospital, Second Military Medical University, ${ }^{2}$ Department of Gastroenterology, \\ Changhai Hospital, Second Military Medical University, Shanghai, ${ }^{3}$ Department of Gastroenterology, PLA Air Force General \\ Hospital, Beijing, China
}

\begin{abstract}
Background and Objectives: Digital image analysis (DIA) has been reported to be a sensitive method to detect pancreatic neoplasms by assessing nuclear DNA content. The aim of the current study was to evaluate the diagnostic performance of DIA for pancreatic malignancy relative to conventional cytology (CC) in patients undergoing EUS-FNA. Patients and Methods: One hundred and forty-two patients with suspected pancreatic malignancy were enrolled prospectively in this study. FNA sample from each patient was evenly divided and assessed by CC and DIA. Findings from histopathology of FNA specimens and a minimum 12-month follow-up were used as the standard. Results: Total 102 out of 142 patients were determined as pancreatic cancer (PC) in this study. The overall sensitivity, specificity, and accuracy of CC were $75 \%, 85 \%$, and $78 \%$, whereas the sensitivity, specificity, and accuracy of DIA were $84 \%, 80 \%$, and $83 \%$, respectively. A combination of CC and DIA (CC/DIA) showed greater sensitivity than CC alone $(92 \% v s .75 \%$, $P<0.01)$, but the specificity was reduced from $85 \%$ to $70 \%(P=0.03)$. Conclusions: In this study, we demonstrated that DIA provided comparable diagnostic performance to $\mathrm{CC}$ in detecting PC. This objective diagnostic method, DIA, emerged as an important supplementary tool to endoscopic biopsy and cytology for diagnosing patients undergoing EUS-FNA with suspected pancreatic malignancy.
\end{abstract}

Key words: Conventional cytology, digital image analysis, EUS-FNA, pancreatic cancer

\begin{tabular}{|l|l|}
\hline \multicolumn{3}{|c|}{ Access this article online } \\
\hline Quick Response Code: & Website: \\
\hline & www.eusjournal.com \\
\hline
\end{tabular}

This is an open access journal, and articles are distributed under the terms of the Creative Commons Attribution-NonCommercial-ShareAlike 4.0 License, which allows others to remix, tweak, and build upon the work non-commercially, as long as appropriate credit is given and the new creations are licensed under the identical terms.

For reprints contact: reprints@medknow.com

How to cite this article: Kong F, Kong X, Zhu J, Sun T, Du Y, Wang K, et al. A prospective comparison of conventional cytology and digital image analysis for the identification of pancreatic malignancy in patients undergoing EUS-FNA. Endosc Ultrasound 2019;8:269-76.

\footnotetext{
Address for correspondence

Prof. Dong Wang, Department of Gastroenterology, Gongli Hospital, Second Military Medical University, Shanghai, China.

Department of Gastroenterology, Changhai Hospital, Second Military Medical University, Shanghai, China.

E-mail: wangdong@hotmail.com

Prof. Zhaoshen Li, Department of Gastroenterology, Gongli Hospital, Second Military Medical University, Shanghai, China.

Department of Gastroenterology, Changhai Hospital, Second Military Medical University, Shanghai, China.

E-mail: zhaoshenli5610@hotmail.com
}

Received: 2018-09-30; Accepted: 2019-02-05; Published online: 2019-05-09 


\section{INTRODUCTION}

EUS-FNA is routinely used to evaluate pancreatic cancer (PC). However, the interpretation of histologic samples of PC is sometimes difficult because of the inflammation, fibrosis, and small tissue fragments within collected samples. Cytological test has been readily used as supplementary tool to EUS-FNA in clinical applications. Conventional cytology (CC) has high specificity, but its sensitivity in diagnosing pancreatobiliary malignancy is highly variable, ranging from $15 \%$ to $90 \% .^{[1-10]}$ The difference of diagnostic sensitivity provided by CC is, at least partially, due to its subjective characteristics. The low diagnostic sensitivity of CC for PC often leaves uncertainty as to the validity of negative interpretation.

Digital image analysis (DIA) is a relatively new technique of cytological analysis that quantifies cellular constituents and analyzes DNA ploidy using spectrophotometric principles. ${ }^{[6,8,11,12]}$ The potential mechanism to explain why the abnormal change of ploidy can contribute to oncogenesis may be that most aneuploidy cells are genetically unstable. ${ }^{[13]}$ DIA has been demonstrated to be an effective diagnostic method in detecting pancreatic malignancy. By endoscopic retrograde cholangiopancreatography (ERCP) cytologic samples, individual DIA test has been reported to provide greater diagnostic sensitivity than $\mathrm{CC}^{\left[{ }^{[4]} \mathrm{A}\right.}$ composite test of CC/DIA was reported to improve the sensitivity for detecting malignant lymph nodes. ${ }^{[6]}$ A sensitivity of $85 \%$ for diagnosing PC was reported from a Mayo Clinic study in the United States. ${ }^{[8]}$ However, deficiency of comparative and prospective evidence leaves the diagnostic benefit of DIA for PC still controversial.

In this study, we conducted a prospective clinical trial with largest sample size up to date to compare the diagnostic performance of DIA with CC for patients undergoing EUS-FNA with suspected pancreatic malignancy.

\section{PATIENTS AND METHODS}

\section{Patients}

This prospective and comparative study was performed in Changhai Hospital between October 2014 and October 2016. The study protocol was approved by the Clinical Research Ethics Committee of Changhai Hospital. Informed consent was obtained from all patients, and all authors had access to the study data and reviewed and approved the final manuscript. This study was registered with ClinicalTrials.gov (NCT02921100).

Consecutive patients undergoing EUS-FNA were invited to participate in our study based on inclusion criteria of (1) an age of 18 years or older with (2) suspected pancreatic malignancy. Patients with the following conditions were excluded: (1) coagulopathy (international normalized ratio >1.5), (2) marked thrombocytopenia (platelet count $<50,000 / \mathrm{ml}$ ), (3) anticoagulant therapy or therapy with antiplatelet agents, and (4) inability to complete follow-up.

A final diagnosis of malignancy was made if there was (1) histopathological confirmation by endoscopic fine-needle biopsy, (2) radiographic evidence from the following 12 months demonstrating involvement of adjacent structures and the presence of distant metastases (hepatic, pulmonary, or bone), or (3) cancer-related death. Cases without a malignant histopathologic diagnosis or without adequate tissue specimens for histopathologic evaluation underwent 12-month follow-up. Confirmation of benign lesions for a patient required the absence of all of the above criteria during the 12-month follow-up [Figure 1]. DIA findings were excluded from the medical records and did not affect patient care.

\section{EUS-FNA}

EUS-FNA processing was performed with a standard 22-gauge needle (Echotip; Cook Medical, Winston-Salem, $\mathrm{NC}$, USA) using $5 \mathrm{ml}$ of negative pressure and standard techniques as previously reported. ${ }^{[14,15]}$ Biopsy specimens were taken from the suspicious areas. Additional passes (up to 7) were obtained with the evaluation of an on-site cytotechnologist until adequate specimens for CC were collected in all cases. The entire cytological specimen was then evenly divided and delivered for DIA and CC testing. After that, a quick re-evaluation of cellular yield for CC was performed. The assessment of tissue specimens and the interpretation of CC and DIA were performed by different gastrointestinal cytopathologists who were completely blinded to the clinical information and alternate data of the patients.

\section{Conventional cytology}

The CC was interpreted independently by two cytopathologists according to CC criteria. Both of the cytopathologists who reviewed the specimens were blinded to the DIA results and follow-up information. 


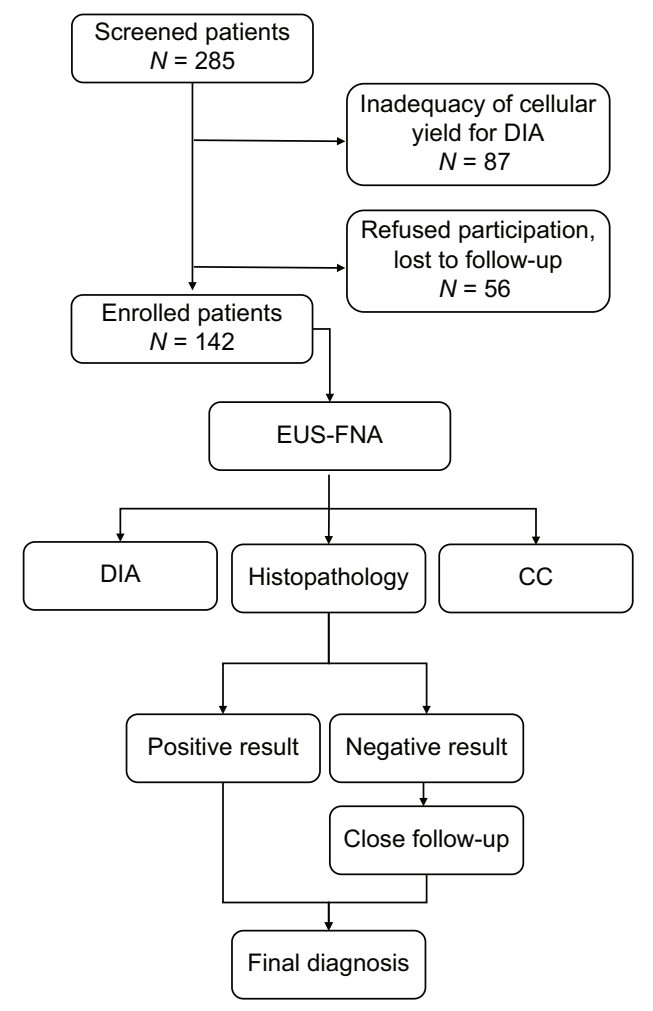

Figure 1. Flowchart of the study

For CC, interpretations were classified into four categories: "benign," "atypical," "suspicious for malignancy," and "malignant." Any disagreement on specimens was referred to a third cytopathologist to provide a final consensus.

\section{Digital image analysis}

DIA is a method of cytologic analysis quantifying cellular constituents using spectrophotometric principles [Figure 2]. Quantification of DNA content, chromatin distribution, and nuclear morphology can be determined and suggested features of malignancy. ThinPrep specimens were prepared as previously described. ${ }^{[16]}$ According to the experience of our center, only specimens with $>500$ cells were prepared for quantification using an image analyzer (Landing Medical High-Tech, Wuhan, Hubei, China) according to the designated protocol. This system automatically loads each slide, scans the area of the ThinPrep deposition, collects images of every object detected, and calculates a set of approximately 120 features for each object. The mean integrated optical density lymphocytes served as an internal standard control. The resulting DNA ploidy value is expressed as a "c" value for chromosome. A DNA ploidy value of $2 \mathrm{c}$ indicates a normal diploid cell, and $4 \mathrm{c}$ indicates a tetraploid cell; $5 \mathrm{c}$ was used in this study as the cutoff to define aneuploidy [Figure 3].

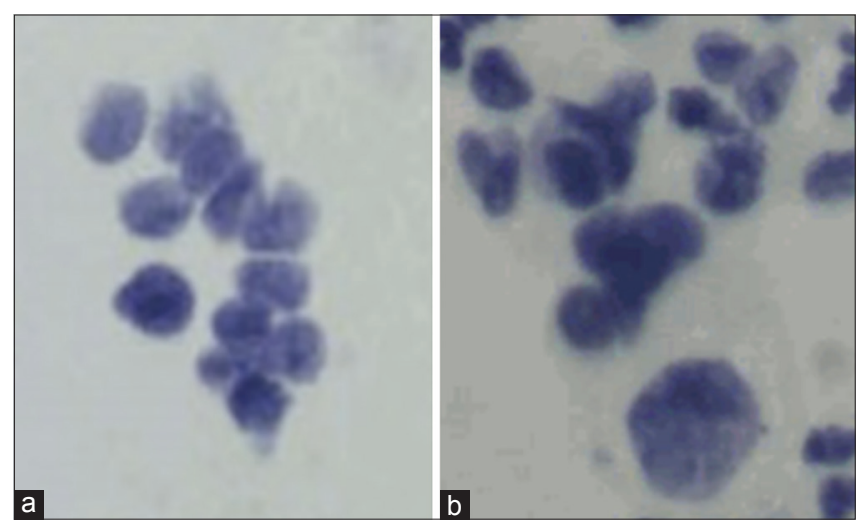

Figure 2. Representative images of FNA specimens during digital image analysis. (a) Feulgen staining during digital image analysis demonstrates a benign sample $(\times 400)$. (b) Feulgen staining during digital image analysis reveals a malignant sample $(\times 400)$. Red arrows indicate typical nucleus

\section{Statistical analysis}

Each participant underwent four cytological measurements to detect pancreatic malignancy: (1) CC, (2) DIA, (3) CC + DIA, and (4) CC/DIA. We classified cytological findings of suspicious for malignancy and malignant as positive results and defined both benign and atypia as negative results. For DIA, a case with at least one 5 c-exceeding event was interpreted as malignant. "CC + DIA" refers to a composite test that defines malignant only when both CC and DIA results were positive and benign when either CC or DIA was negative. "CC/DIA" was considered malignant when either of the tests (CC or DIA) reported positive results and was considered benign only when both tests were reported negative. Due to the difficulty in obtaining adequate tissue specimens to make a histopathologic diagnosis during FNA, all of the patients without a confirmed malignant histopathologic interpretation were followed postprocedurally by telephone contact to ascertain the final diagnosis, vital status, and cause of death.

The accuracy, sensitivity, specificity, positive predictive value, negative predictive value, and 95\% confidence interval of each individual or composite test were estimated and compared using the McNemar test. We used statistical software JMP version 10.0 (SAS Institute Inc., Cary, NC, USA). $P<0.05$ was considered statistically significant.

\section{RESULTS}

\section{Baseline characteristics}

One hundred and forty-two patients with suspicious pancreatic malignancy were enrolled in this study 
Kong, et al.: DIA and CC for pancreatic cancer

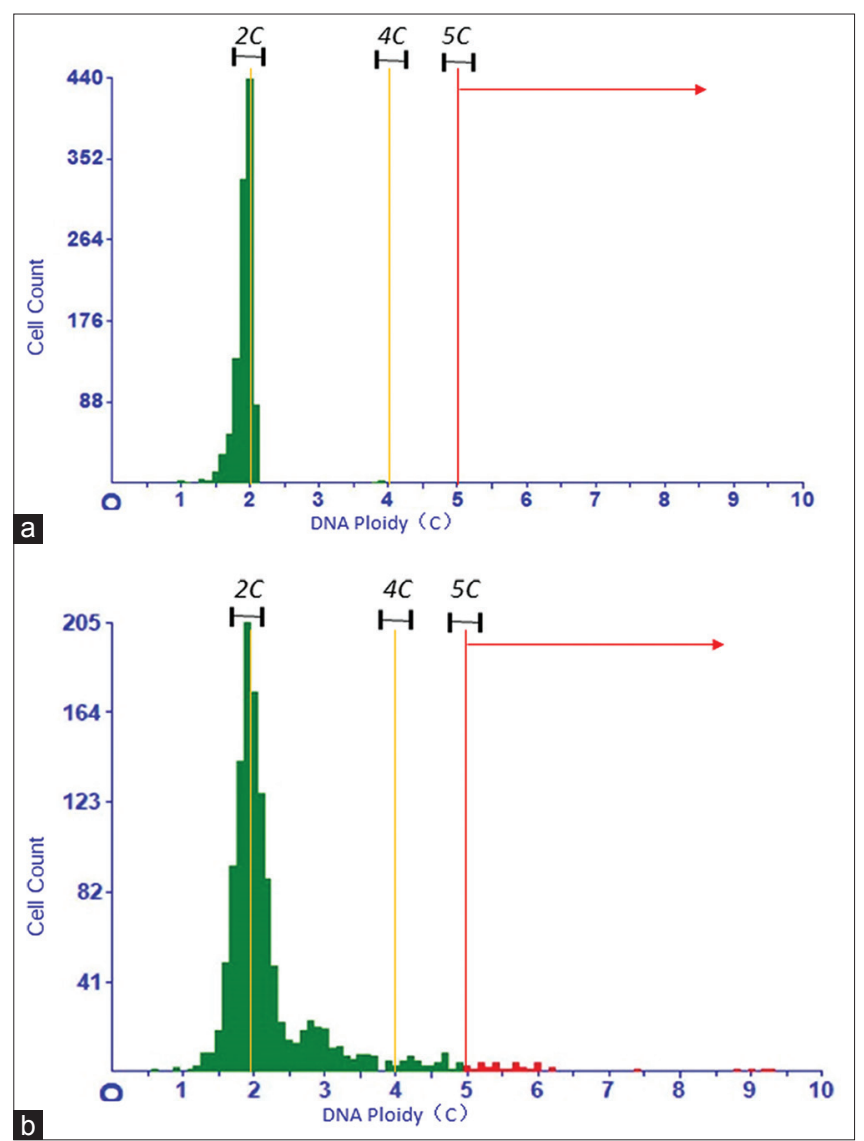

Figure 3. DNA histograms showing cell distributions based on nuclear DNA content. (a) Benign specimen. (b) Malignant specimen. 2c, cells in the diploid range; $4 \mathrm{c}$, cells in the tetraploid range. $5 \mathrm{c}$ was used in this study as the cutoff to define aneuploidy. Any cases with at least one $5 c$-exceeding event were interpreted as malignant cases

according to the study protocol (median age, 62 years; range, 18-80 years; 94 males/48 females). Diagnoses of PC were made in 102 patients (72\%). Among the 102 patients, 38 patients had a diagnosis confirmed by endoscopic FNA on the pancreatic lesions, and 64 patients were confirmed by clinical criteria after 12-month follow-up. Among malignant cases with pathohistological confirmation, adenocarcinoma was the most common pathological type of PC (26/38, 68\%), while the nonadenocarcinoma masses accounted for $32 \%$ in total $(12 / 38)$. The pancreatic masses were mainly found in the head $(68 / 142)$, followed by the body (22/142), tail (22/142), and whole organ (20/142) of the pancreas [Table 1].

\section{Performance characteristics}

When considering all patients with the use of gold standard, the sensitivity, specificity, and accuracy of CC were $75 \%, 85 \%$, and $78 \%$, respectively, whereas the sensitivity, specificity, and accuracy of DIA were $84 \%$, $80 \%$, and $83 \%$, respectively. Although there was a $9 \%$
Table 1. Baseline characteristics of patients

\begin{tabular}{lcc}
\hline & $n$ (total=142) & Percentage \\
\hline Age/years & & \\
Median & 62 & \\
Range & $18-80$ & \\
Sex & & \\
Male & 94 & $66(94 / 142)$ \\
Female & 48 & $34(48 / 142)$ \\
Diagnoses & & \\
Malignant & 102 & $72(102 / 142)$ \\
$\quad$ With pathohistological & 38 & $37(38 / 102)$ \\
$\quad$ confirmation & & \\
$\quad$ Adenocarcinoma & 26 & $68(26 / 38)$ \\
$\quad$ Nonadenocarcinoma & 12 & $32(12 / 38)$ \\
$\quad$ With clinical confirmation & 64 & $63(64 / 102)$ \\
Benign & 40 & $28(40 / 142)$ \\
Location & & \\
Uncinate process & $10(8 / 2)^{\mathrm{a}}$ & $7(10 / 142)$ \\
Head & $68(50 / 18)^{\mathrm{a}}$ & $48(68 / 142)$ \\
Body & $22(12 / 10)^{\mathrm{a}}$ & $15(22 / 142)$ \\
Tail & $22(20 / 2)^{\mathrm{a}}$ & $15(22 / 142)$ \\
Diffuse enlargement & $20(12 / 8)^{\mathrm{a}}$ & $14(20 / 142)$ \\
\hline aalignant cases/benign cases & &
\end{tabular}

and $5 \%$ increase in sensitivity and accuracy provided by DIA, these changes did not exhibit statistical significance $(P=0.08$ and $P=0.23$, respectively). The specificity of "CC + DIA" was not significantly superior to CC alone ( $95 \%$ vs. $85 \%, P=0.13)$. Of note, the sensitivity and accuracy of the composite test CC/DIA were significantly higher than CC alone (92\% vs. $75 \%, P<0.01 ; 86 \%$ vs. $78 \%, P=0.02$ ), but the specificity decreased significantly from $85 \%$ to $70 \%(P=0.03)$ [Table 2].

Performance of digital image analysis and conventional cytology for the diagnosis of different types of pancreatic cancers

Among all of the patients enrolled with pathologic confirmation of malignancy $(n=38)$, adenocarcinoma accounted for $68 \%(n=26)$. Other cancer types included squamous cell carcinoma $(n=5)$, neuroendocrine tumor $(n=4)$, and acinar cell carcinoma $(n=3)$. For these with a diagnosis of cancer based on pathology from FNA specimens, the sensitivity of CC, DIA, CC + DIA, and CC/DIA were $84 \%, 94 \%, 79 \%$, and $100 \%$, respectively. Only CC/DIA increased the sensitivity significantly $(P<0.01)$. For adenocarcinoma, CC, DIA, CC + DIA, and CC/DIA showed a sensitivity of $84 \%, 92 \%, 77 \%$, and $100 \%$, respectively. For the other cancer types, CC, DIA, CC + DIA, and CC/DIA had a sensitivity of $83 \%, 100 \%, 83 \%$, and $100 \%$, respectively. A trend of improved diagnostic 
sensitivity was demonstrated provided by DIA compared with CC $(P=0.06)$ [Table 3]. In addition, neither of the individual tests nor the composite tests provided significantly different diagnostic sensitivity for adenocarcinoma compared with that of the same test(s) in patients for nonadenocarcinoma tumor types.

The remaining 104 patients were diagnosed by clinical evidence or 12-month follow-up information. The diagnostic sensitivity of CC, DIA, CC + DIA, and CC/DIA for this cohort was $69 \%, 78 \%, 59 \%$, and $87 \%$, respectively. However, the observed advantage of sensitivity delivered by DIA and CC/DIA was not statistically significant $(P=0.11$ and $P=0.05$, respectively). For patients with a clinical diagnosis of malignancy $(n=64), 58$ obtained unresectable lesions. Total 42 had radiographic tumor progression, and 22 without radiographic advance died from PC within 12-month follow-up.

Performance of digital image analysis and conventional cytology for the diagnosis of pancreatic cancer by different location

By location, the pancreatic masses were classified into groups of uncinate process, head, body, tail, and diffuse occupancy [Table 4]. The accuracy, sensitivity, and specificity of CC showed comparable performance characteristics when compared with DIA, CC + DIA, and CC/DIA. For the 68 lesions located in the head of the pancreas, DIA had $56(83 \%)$ accurate diagnoses and CC had $46(68 \%)$ accurate diagnoses. Thus, DIA resulted in an increase in accuracy by $14 \%$; however, the statistical difference was borderline $(P=0.05)$.

Effect of cell number on the diagnostic performance of digital image analysis

To explore the effect of cell number on the diagnostic efficacy of DIA, we increased the cutoff number of cells for DIA from 500 to 1000, 1500, or 2000 [Table 5]. DIA produced a better sensitivity and accuracy with the increase in cell number, while the specificity remained at a comparable level. However, the enhanced diagnostic performance seemed to reach a plateau when the cutoff value increased to 1500 . When the cutoff increased to 1000 , cases meeting this including criterion decreased to $<80 \%$, which would restrict the clinical application of DIA.

\section{DISCUSSION}

In this prospective comparison, DIA showed comparable test characteristics relative to $\mathrm{CC}$ in

Table 2. Performance characteristics of pancreatic FNA: Malignant plus benign

\begin{tabular}{|c|c|c|c|c|}
\hline & $\mathrm{CC}$ & DIA & $C C+D I A$ & CC/DIA \\
\hline Sensitivity & $75(0.65-0.83)$ & $84(0.76-0.90)$ & 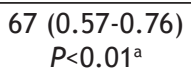 & 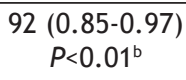 \\
\hline Specificity & $85(0.65-0.82)$ & $80(0.64-0.99)$ & $95(0.83-0.99)$ & $\begin{array}{c}70(0.53-0.83) \\
P=0.03^{\mathrm{a}}\end{array}$ \\
\hline PPV & $93(0.85-0.98)$ & $92(0.84-0.97)$ & $97(0.90-1)$ & $89(0.81-0.94)$ \\
\hline NPV & $43(0.43-0.70)$ & $67(0.52-0.80)$ & $53(0.41-0.65)$ & $78(0.61-0.90)$ \\
\hline Accuracy & $78(0.72-0.88)$ & $83(0.74-0.90)$ & $75(0.74-0.88)$ & $\begin{array}{c}86(0.72-0.90) \\
P=0.02^{b}\end{array}$ \\
\hline
\end{tabular}

aStatistically significant $(P<0.05)$ decrease relative to CC, bStatistically significant $(P<0.05)$ increase relative to CC. All values are expressed as percentage $(95 \% \mathrm{Cl})$. CC: Conventional cytology, DIA: Digital image analysis, $\mathrm{Cl}$ : Confidence interval, PPV: Positive predictive value, NPV: Negative predictive value

Table 3. Sensitivity based on tumor type for pancreatic FNA: Adenocarcinoma plus nonadenocarcinoma

\begin{tabular}{lcccc}
\hline & CC & DIA & CC + DIA & CC/DIA \\
\hline Adenocarcinoma $(n=26)$ & $84(0.62-0.96)$ & $92(0.75-0.99)$ & $77(0.56-0.91)$ & $100(-)$ \\
Nonadenocarcinoma $(n=12)$ & $83(0.52-0.98)$ & $100(0.74-1)$ & $83(0.52-0.98)$ & $100(-)$ \\
\hline
\end{tabular}

CC: Conventional cytology, DIA: Digital image analysis

Table 4. Sensitivity based on tumor location for pancreatic FNA

\begin{tabular}{lcccc}
\hline & CC & DIA & CC+DIA & CC/DIA \\
\hline Uncinate process $(n=10)$ & $25(0.03-0.65)$ & $75(0.35-0.97)$ & $25(0.03-0.65)$ & $75(0.35-0.97)$ \\
Head $(n=68)$ & $68(0.53-0.81)$ & $84(0.71-0.93)$ & $60(0.45-0.74)$ & $92(0.81-0.97)$ \\
Body $(n=22)$ & $67(0.35-0.91)$ & $83(0.52-0.98)$ & $67(0.34-0.90)$ & $83(0.52-0.98)$ \\
Tail $(n=22)$ & $100(0.832-1)$ & $90(0.68-0.99)$ & $90(0.68-0.99)$ & $100(0.83-1)$ \\
Diffuse enlargement $(n=20)$ & $100(0.735-1)$ & $75(0.35-0.97)$ & $83(0.52-0.98)$ & $100(0.74-1)$ \\
\hline
\end{tabular}

CC: Conventional cytology, DIA: Digital image analysis 
Kong, et al.: DIA and CC for pancreatic cancer

Table 5. Performance characteristics of digital image analysis for pancreatic FNA based on cell number

\begin{tabular}{lcccc}
\hline & $500(n=142)$ & $1000(n=112)$ & $1500(n=96)$ & $2000(n=78)$ \\
\hline Sensitivity & $84(0.76-0.96)$ & $91(0.83-0.96)$ & $93(0.86-0.97)$ & $94(0.85-0.98)$ \\
Specificity & $80(0.64-0.99)$ & $82(0.60-0.95)$ & $86(0.57-0.97)$ & $83(0.52-0.98)$ \\
PPV & $92(0.84-0.97)$ & $95(0.89-0.99)$ & $97(0.91-1.00)$ & $97(0.90-1.00)$ \\
NPV & $67(0.52-0.80)$ & $69(0.48-0.86)$ & $67(0.41-0.87)$ & $71(0.42-0.92)$ \\
Accuracy & $83(0.74-0.90)$ & $89(0.76-0.97)$ & $92(0.78-1.00)$ & $93(0.76-1.00)$ \\
\hline
\end{tabular}

PPV: Positive predictive value, NPV: Negative predictive value

detecting PC. However, when using the composite tests, we found a significantly improved sensitivity and comparable specificity by CC/DIA relative to CC alone. Unexpectedly, we did not achieve better specificity when we used the composite test "CC + DIA" compared with CC alone. The test characteristics of DIA has been reported in previous studies for the detection of malignant pancreatobiliary strictures..$^{[4,7,8,12,17]}$ By ERCP and brush cytology, the sensitivity of CC was reported to range from $15 \%$ to $38 \%$, and the sensitivity of DIA ranged from $39 \%$ to $62 \%$. As expected, both DIA and CC in this study from FNA samples showed better performance than previously reported results from ERCP and brush cytology. The most plausible explanation is that tumors that affect the pancreatobiliary tract are often fibrotic and may not invade the ductal epithelium, especially when arising from surrounding structures or metastasizing from distant sites. As a result, tissue-sampling techniques directly targeting the lesion areas may produce a cellular specimen with greater diagnostic significance.

PC is a solid tumor with considerable proportion of fibrosis and obtaining adequate tissue specimens by FNA is technically difficult. Therefore, negative results by histopathology from FNA specimens do not definitively exclude a malignant diagnosis. To evaluate the true diagnostic power of CC and DIA, we followed all of the patients postprocedurally without a malignant interpretation confirmed by histopathology, which was also included as part of gold standard. Within this population, CC/DIA still showed the highest sensitivity, and the enhancement relative to CC was statistically significant.

The maximum sensitivity of $\mathrm{CC}$ can be influenced by the different criteria for malignant cases. To provide a higher sensitivity, we recalculated the data by including severely atypia cases as positive results, and we observed a $10 \%$ increase of sensitivity for CC (data not shown). However, the enhanced sensitivity did not exhibit statistically superior performance relative to DIA in the overall population. We observed that the specificity reported in this study was relatively low. One explanation is that we included "suspicious for malignancy" as a positive cytological interpretation. Since CC is a technology highly dependent on the skill of the interpreting cytologist, another explanation may be that the cytologists in our center may not have been as conservative in diagnosing cancer as cytologists from other centers. In addition, some malignant tumors grew slowly during our follow-up period and might be falsely diagnosed as benign cases. Based on the progressive nature of $\mathrm{PC}$ and the strict follow-up context, we suspect that this proportion must be small.

$5 \mathrm{c}$ is a cutoff used for aneuploidy by many authors, ${ }^{[11,18]}$ whereas other authors favor $9 \mathrm{c}^{[19,20]}$ or a DNA index (DI) beyond 1.10. ${ }^{[6]}$ DNA content exceeding $5 \mathrm{c}$ was one of the frequently used parameters referred by DIA to replace the DI and can be used to further analyze the degree of hyperdiploidy. The findings in our study established that $5 c$ was an effective cutoff value to determine pancreatic malignancy relative to CC. However, this approach biased the diagnostic sensitivity of DIA for the exclusion of aneuploidy within $5 \mathrm{c}$. Therefore, further studies should be conducted using different parameters to compare the diagnostic performance of DIA and CC, and an enhanced diagnostic performance of DIA should be expected.

In previous studies, the cutoffs of cell numbers chosen for DIA analysis were variable, ranging from dozens to hundreds. ${ }^{[6,7,12,18]}$ To determine the optimal cellularity, we tested the effect of cellularity on the performance of DNA ploidy. Unlike previous protocols that tested $<200$ selected cells, our DIA system simultaneously analyzed up to 3000 cells to make a final determination. As expected, the larger the number of cells scanned, the higher the performance was optimized. However, when the cutoff increased to 1000 , the proportion of cases meeting this requirement decreased below $80 \%$, which would limit the application of DIA in clinical practice. Levy et al. 
reported a decreased sensitivity of DIA to detect pancreatic malignancy relative to CC. In that study, only one-quarter of the cytological specimen from FNA was assigned for DIA. ${ }^{[6]}$ Since no enhanced sensitivity of CC was found using pass-by-pass analysis in that study, we speculate that the addition of cytological specimens may have little effect on the performance of CC but attenuates the diagnostic power of DIA.

DIA is highly automated and not as labor intensive as it was previously. The major work of a technician at present is focused on excluding all of the artifacts from the $>5 \mathrm{c}$ cell galleries. With the development of this technique, DIA is not as costly as before in our center ( $<80$ dollars/each test). The comparable diagnostic performance of DIA identified in this study provides possibility of the replacement of CC with DIA during EUS-FNA. A composite CC/DIA may increase diagnostic sensitivity to detect pancreatic malignancy. However, results obtained in this study still need to be validated by more clinical trials. At present, the utilization of DIA in endoscopic centers without support from experienced cytopathologists may be recommended.

The limitations of our study include the lack of surgical pathology in many patients and the considerable proportion of patients who were finally diagnosed by follow-up information. The use of only enrolled patients confirmed on the basis of surgical pathology could exclude randomized patients from our analysis, but this approach is neither plausible nor in keeping with standard practices. In our center, most patients undergoing EUS-FNA test carried unresectable lesions at the time of diagnosis. Surgical tissue specimens were hardly to be obtained to review for these groups of patients. However, we considered that the patients we included in this study may be more similar to the patients encountered in the routine clinical practice.

\section{CONCLUSIONS}

This study demonstrated that DIA can provide comparable diagnostic performance to $\mathrm{CC}$ in diagnosing pancreatic malignancy and higher sensitivity for patients without a malignant histopathological diagnosis from endoscopic biopsy. Using a composite CC/DIA test, diagnostic sensitivity can be greatly enhanced compared with that of CC alone. DIA, based on objective findings, seems to be effective method complementary to endoscopic biopsy, and we recommend further studies to valid its clinical value in detecting PC during EUS-FNA.

\section{Financial support and sponsorship}

This project was funded by medical Discipline Construction Project of Pudong New Area Commission of Health and Family Planning (Grant No.PWYgf2018-01) and National Natural Science Foundation of China (Grant No. 81402425).

\section{Conflicts of interest}

There are no conflicts of interest.

\section{REFERENCES}

1. Erickson RA. EUS-guided FNA. Gastrointest Endosc 2004;60:267-79.

2. Gleeson FC, Kipp BR, Caudill JL, et al. False positive endoscopic ultrasound fine needle aspiration cytology: Incidence and risk factors. Gut 2010;59:586-93.

3. Vincent A, Herman J, Schulick R, et al. Pancreatic cancer. Lancet 2011;378:607-20.

4. Baron TH, Harewood GC, Rumalla A, et al. A prospective comparison of digital image analysis and routine cytology for the identification of malignancy in biliary tract strictures. Clin Gastroenterol Hepatol 2004;2:214-9.

5. Levy MJ, Clain JE, Clayton A, et al. Preliminary experience comparing routine cytology results with the composite results of digital image analysis and fluorescence in situ hybridization in patients undergoing EUS-guided FNA. Gastrointest Endosc 2007;66:483-90.

6. Levy MJ, Oberg TN, Campion MB, et al. Comparison of methods to detect neoplasia in patients undergoing endoscopic ultrasound-guided fine-needle aspiration. Gastroenterology 2012;142:1112-21.e2.

7. Moreno Luna LE, Kipp B, Halling KC, et al. Advanced cytologic techniques for the detection of malignant pancreatobiliary strictures. Gastroenterology 2006;131:1064-72.

8. Rumalla A, Baron TH, Leontovich $\mathrm{O}$, et al. Improved diagnostic yield of endoscopic biliary brush cytology by digital image analysis. Mayo Clin Proc 2001;76:29-33.

9. Ryan ME, Baldauf MC. Comparison of flow cytometry for DNA content and brush cytology for detection of malignancy in pancreaticobiliary strictures. Gastrointest Endosc 1994;40:133-9.

10. Lee YN, Moon JH, Kim HK, et al. Core biopsy needle versus standard aspiration needle for endoscopic ultrasound-guided sampling of solid pancreatic masses: A randomized parallel-group study. Endoscopy 2014;46:1056-62.

11. Guillaud M, Benedet JL, Cantor SB, et al. DNA ploidy compared with human papilloma virus testing (Hybrid capture II) and conventional cervical cytology as a primary screening test for cervical high-grade lesions and cancer in 1555 patients with biopsy confirmation. Cancer 2006;107:309-18.

12. Osterheld MC, Andrejevic Blant S, Caron L, et al. Digital image DNA cytometry: A useful tool for the evaluation of malignancy in biliary strictures. Cell Oncol 2005;27:255-60.

13. Senovilla L, Vitale I, Martins I, et al. An immunosurveillance mechanism controls cancer cell ploidy. Science 2012;337:1678-84.

14. Chang KJ, Katz KD, Durbin TE, et al. Endoscopic ultrasound-guided fine-needle aspiration. Gastrointest Endosc 1994;40:694-9.

15. Wiersema MJ, Vilmann P, Giovannini M, et al. Endosonography-guided fine-needle aspiration biopsy: Diagnostic accuracy and complication assessment. Gastroenterology 1997;112:1087-95.

16. Sun XR, Wang J, Garner D, et al. Detection of cervical cancer and high 
grade neoplastic lesions by a combination of liquid-based sampling preparation and DNA measurements using automated image cytometry. Cell Oncol 2005;27:33-41.

17. Krishnamurthy S, Katz RL, Shumate A, et al. DNA image analysis combined with routine cytology improves diagnostic sensitivity of common bile duct brushing. Cancer 2001;93:229-35.

18. Pradhan M, Abeler VM, Danielsen HE, et al. Image cytometry DNA ploidy correlates with histological subtypes in endometrial carcinomas.
Mod Pathol 2006;19:1227-35.

19. Lorenzato M, Clavel C, Masure M, et al. DNA image cytometry and human papillomavirus (HPV) detection help to select smears at high risk of high-grade cervical lesions. J Pathol 2001;194:171-6.

20. Bollmann R, Méhes G, Speich N, et al. Aberrant, highly hyperdiploid cells in human papillomavirus-positive, abnormal cytologic samples are associated with progressive lesions of the uterine cervix. Cancer 2005;105:96-100. 